\title{
The targeted podocyte
}

\author{
Agnes B. Fogo \\ Department of Pathology, Vanderbilt University Medical Center, Nashville, Tennessee, USA.
}

\begin{abstract}
The podocyte plays a key role both in maintenance of the glomerular filtration barrier and in glomerular structural integrity. Podocyte injury and loss contribute to proteinuria and progressive sclerosis. Inhibitors of mammalian target of rapamycin (mTOR) have variably decreased or caused proteinuria and sclerosis in human disease and experimental settings. In this issue of the JCI, two interesting studies of podocyte-specific manipulation of the mTOR system shed light on the complexity of this pathway in the podocyte.
\end{abstract}

\section{Introduction to the podocyte}

The podocyte has become a crucial focus as a target for interventions in chronic kidney disease (CKD) due to its key roles in regulating glomerular permeability and maintaining glomerular structure through interactions with other glomerular parenchymal cells, including endothelial cells. Various slit diaphragm and cytoskeletal proteins contribute to the maintenance of podocyte permeability functions, and podocyte secretion of growth factors such as VEGF is necessary for endothelial cell survival. The podocyte itself also expresses numerous receptors and responds to many growth factors and metabolic products implicated in progressive kidney diseases (1). However, podocytes have limited proliferative capacity, and when glomerular growth and hemodynamic stresses exceed the ability of podocytes to undergo hypertrophy, they become irreversibly injured or detach. The resulting denuded underlying glomerular basement membrane then may give rise to adhesions, a likely nidus for progressive glomerulosclerosis. Niche stem cells in a parietal location, i.e., lining Bowman's capsule, can transition to a visceral location overlying the glomerular tuft (Figure 1 and ref. 2). Podocyte injury with foot process effacement precedes overt sclerosis when focal segmental glomerulosclerosis (FSGS) recurs in kidney transplant recipients. In these patients, we have observed epithelial cells expressing specific parietal epithelial markers, rather than podocyte markers, overlying the glomerular tuft (3). In collapsing glomerulopathy, a variant of FSGS with a particularly bad prognosis, the

Conflict of interest: The author has declared that no conflict of interest exists.

Citation for this article: J Clin Invest. doi:10.1172/ JCI57935. proliferating visceral epithelial cells also display a parietal phenotype (4). Whether such parietal replacement of lost or injured podocytes is restorative, profibrotic, or both, depending on the balance of other injuries, has not been determined.

\section{Kidney injury and mTOR}

Kidney scarring is potentially reversible, as demonstrated in both human diabetic nephropathy and various animal models of CKD. Some therapeutic interventions that mediated regression specifically targeted restoration of podocyte number (5-7). Clearly, protection of podocytes from injurious factors and restoration of podocyte numbers after injury constitute a major challenge in the successful treatment of progressive CKD. In this issue of the JCI, there is a doublet of articles by Gödel et al. (8) and Inoki et al. (9) that demonstrate the importance of the mTOR pathway in podocytes. The data generated may have important implications for understanding the mechanisms of progressive podocyte injury.

mTOR is a widely expressed protein kinase that mediates its functions in two complexes, mTOR complex 1 (mTORC1) and mTORC2. In addition to mTOR, mTORC1 and mTORC2 include the key mTOR (Raptor) and rapamycin-insensitive companion of mTOR (Rictor), respectively. The net consequences of mTOR activity for the podocyte and glomerular disease depend on the balance of these complexes (Figure 2). The downstream effects of mTORCs also may differ in parenchymal versus inflammatory cells. mTORC1 regulates proliferation and autophagy in response to various nutrients. It is activated by the small GTPases Rheb and Rags, and this activation is in turn suppressed by the action of the tuberous sclerosis complex molecules regulatory-associated protein of
(TSC). mTORC1 is potently inhibited by the immunosuppressive drug rapamycin. mTORC 2 controls cell survival and modulates the cytoskeleton and is largely rapamycin insensitive; its activation results in phosphorylation of AKT and PKC (10).

Inhibitors of the mTOR pathway are being intensively investigated as treatments for renal cancers and cystic kidney disease. mTOR inhibition has also been suggested as a treatment in CKD, but results in various settings of human and experimental disease have been variable. Rapamycin ameliorated the development of injury in some experimental models of diabetic nephropathy but not others (10). However, neither streptozotocin injection nor $d b / d b$ mice are models that directly mirror human diabetic nephropathy, in that they manifest only mild mesangial expansion, proteinuria, and glomerular basement membrane (GBM) thickening, and they are not characterized by progressive decline in glomerular filtration rate or nodular glomerulosclerosis and interstitial fibrosis, features characteristic of human diabetic nephropathy. Although rapamycin decreased proteinuria in some patients with FSGS, its use was associated with de novo development of FSGS lesions and proteinuria in some kidney transplant recipients and in some diabetic patients receiving pancreatic transplants (but who retained their own kidneys) (10). It is possible that mTOR inhibition was injurious in these states because glomerular enlargement and presumably podocyte hypertrophy already existed, whether due to the diabetic state or compensatory hypertrophy in the transplant patient with only one kidney. However, the exact mechanisms, whether direct glomerular or immunomodulatory effects, and net results of mTOR inhibition have remained muddled, preventing establishment of a rational basis for targeting $\mathrm{mTOR}$ in CKD.

\section{Podocyte-specific mTORC actions}

The elegant studies by Gödel et al. (8) and Inoki et al. (9) provide more in-depth understanding of the podocyte-specific consequences of mTORC activity and pave the way for further development of targeted and disease-specific interven- 
A Healthy glomerulus

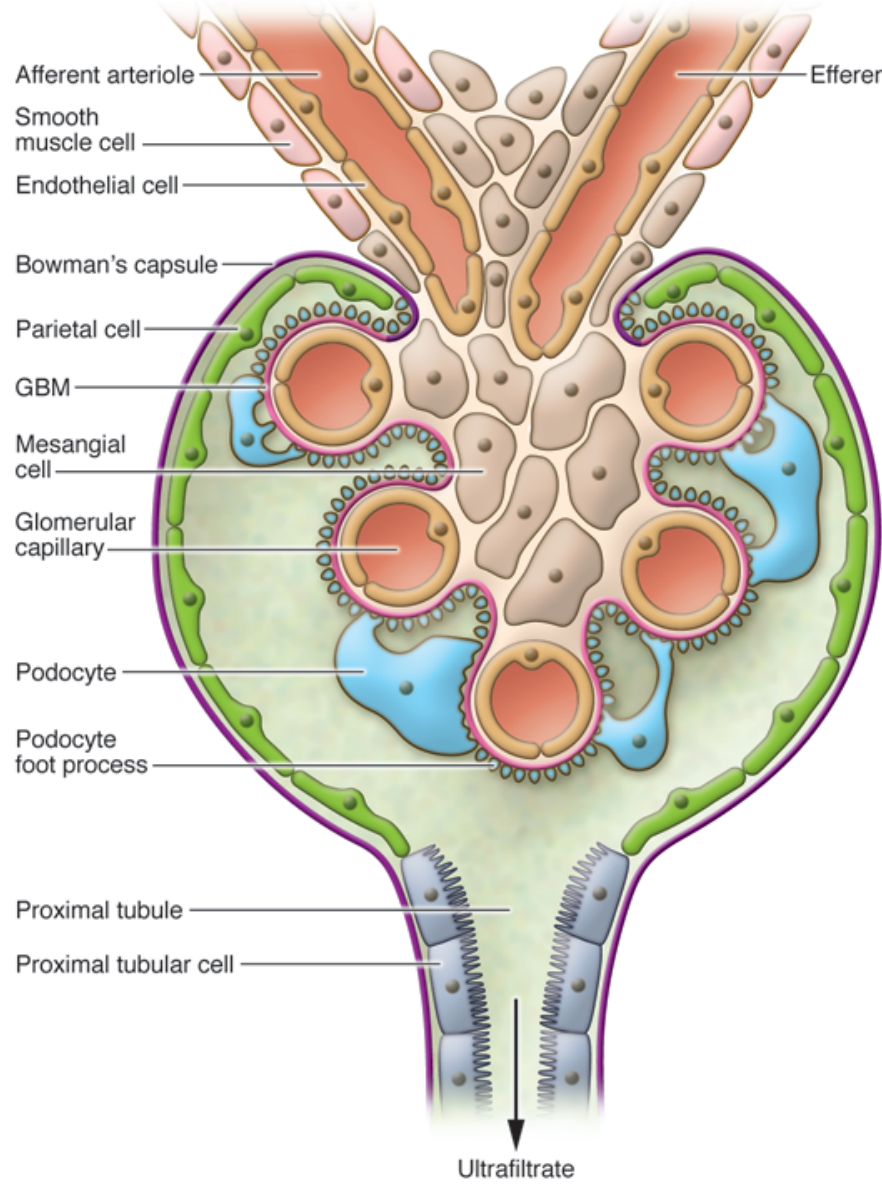

B After podocyte injury
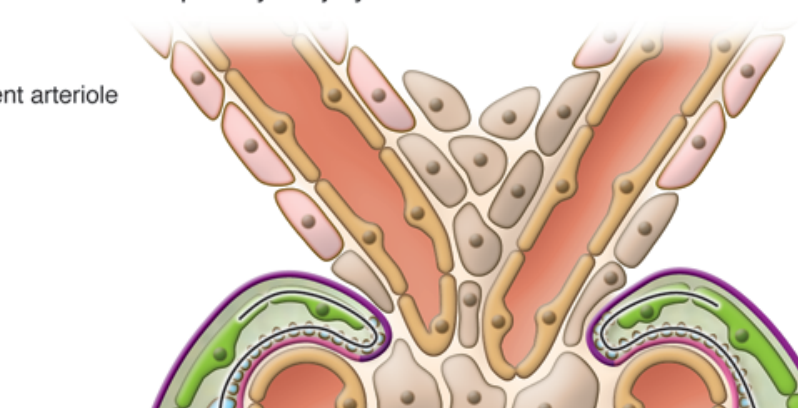

Podocyte detachment

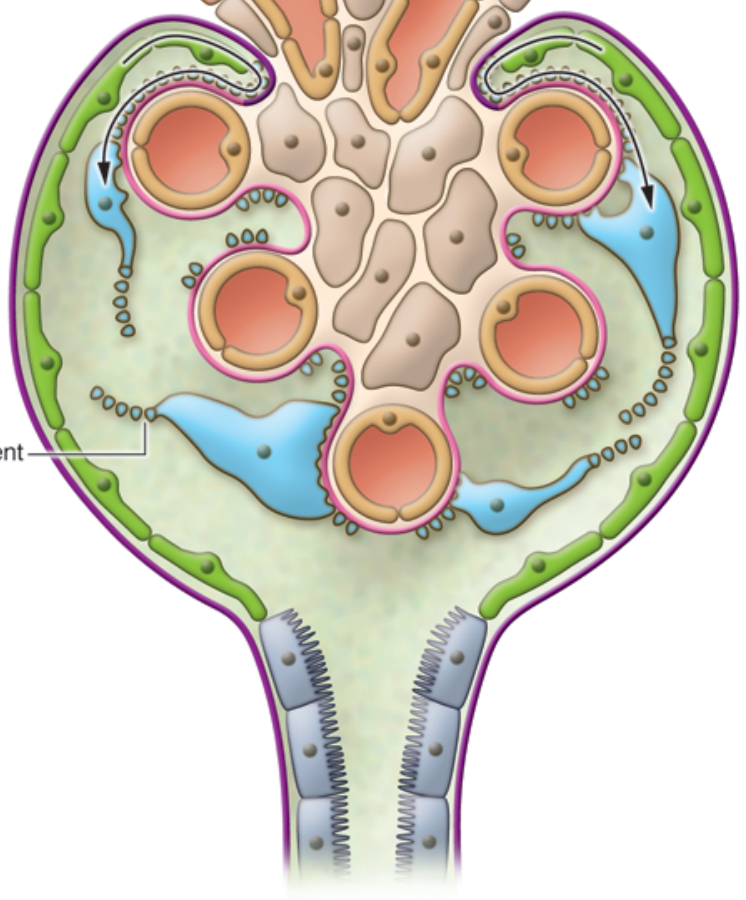

\section{Figure 1}

Glomerular parietal epithelial cells and the injury response. (A) The glomerular tuft is surrounded by Bowman's capsule, which is lined by parietal epithelial cells. The highly specialized podocyte covers the glomerular capillary tuft and is key for maintaining glomerular structure and permeability barrier function. Podocytes have limited proliferative capacity. (B) After podocyte injury and detachment, parietal epithelial cells may migrate along Bowman's capsule and transition to the tuft to areas of injury. Whether these parietal cells are reparative or ultimately promote sclerosis after injury remains unknown.

tions. The authors selectively modulated mTORC1 and mTORC2 activity in mice by deleting Raptor and Rictor, respectively, in podocytes at various stages of disease in models of type 1 and type 2 diabetes and compared the expression of mTOR pathway signaling molecules in these models with their expression in early human diabetic nephropathy. Although mTOR activity was increased in human kidneys with diabetic nephropathy, genetic deletion of podocyte mTORC1 activity (via Raptor deletion) induced proteinuria and glomerular lesions in mice $(8,9)$, and concomitant Rictor deletion (which eliminates mTORC2 activity) exacerbated this phenotype (8). However, activation of mTORC1 specifically in podocytes by genetic deletion of the upstream negative regulator
Tsc1 also resulted in injury (9). These mice developed thickened GBM, proteinuria, and mesangial expansions in addition to podocyte loss. The observation that the ensuing lesions were not restricted to the podocyte demonstrates the importance of crosstalk and interplay between glomerular cells. Podocytes in the Tsc1 $1^{-/-}$mice showed abnormal slit diaphragms and hypertrophy as well as a more mesenchymal phenotype, conditions that are postulated to be pivotal for proteinuria and fibrosis, respectively. Whether these epithelial cells overlying the glomerular tuft retained podocyte markers or included cells with parietal phenotype derived from parietal stem cells remains unknown. Similar epithelial-mesenchymal transition has been observed in podocytes in human diabetic nephropathy, IgA nephropathy, and lupus nephritis, with de novo podocyte expression of fibroblast-specific protein-1 (11). It remains unknown whether mTORC1 is activated in podocytes in all these human conditions, and whether inhibition of its activity could restore normal podocyte phenotype.

Both sets of researchers then induced a perhaps more pathophysiological manipulation of mTOR, namely heterozygous deletion in podocytes of Raptor, resulting in decreased but not absent podocyte mTORC1 activity $(8,9)$. These mice were protected against mild diabetic glomerular injury. The altered mTORC1 activity induced in these experiments also resulted in increased ER stress, and when this stress was decreased by a chemical chaperone, podocyte loss was prevented, although 


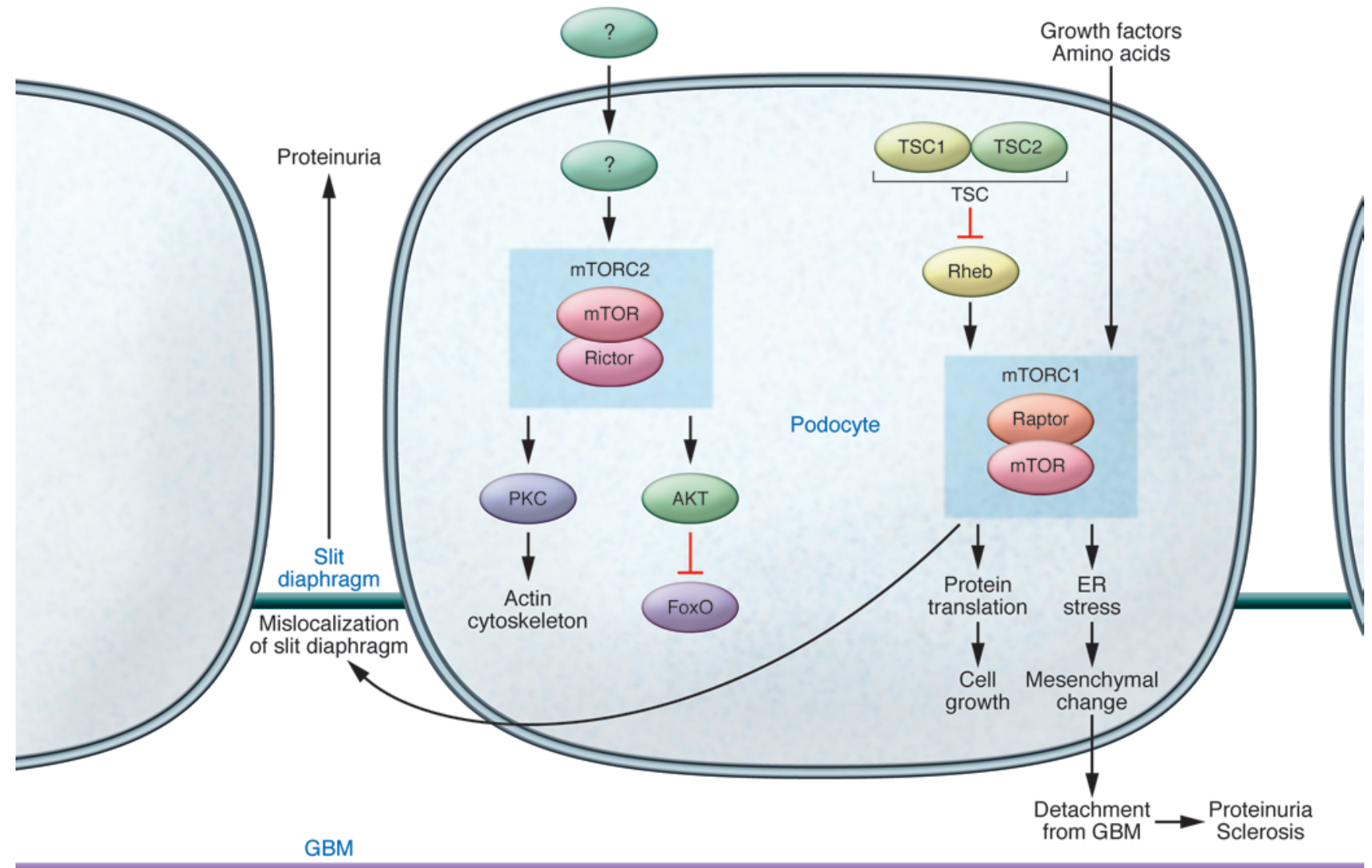

Figure 2

Podocyte maintenance is dependent on a fine-tuned balance of mTORC1 and mTORC2 activity. mTORC1 signaling is normally activated by nutrients such as amino acids and growth factors. The primary signals for mTORC2 activation are unknown. Activation of mTORC1, as occurs in diabetic nephropathy, results in effaced foot processes and proteinuria and also is associated with mesangial expansion. mTORC1 activation may also result in transition of the podocyte to a more mesenchymal phenotype, resulting in loss of adhesion to the underlying GBM and detachment, further promoting proteinuria and ultimately sclerosis. Rapamycin has an immediate inhibitory effect on mTORC1 and with chronic use may also, through unknown mechanisms, inhibit mTORC2. Inhibition of mTORC1, especially during development or other physiologic or pathophysiologic growth, may also cause podocyte injury $(8,9)$. Added inhibition of mTORC2 activation causes more severe podocyte injury, sclerosis, and proteinuria (8), illustrating the dependence of normal podocyte function and structure on balance of the two complexes.

nephrin mislocalization persisted. Such ER stress may be a common effector mechanism of podocyte injury. Podocyte injury and ER stress have also been demonstrated in aging-related sclerosis, with amelioration of injury by a PPAR $\gamma$ agonist linked to decreased mitochondrial injury and oxidative stress (12). It will be interesting to know whether mTOR inhibition could be synergistic with other interventions that decrease ER stress, and possibly even effect reversal of age-related degeneration.

The timing and effects of rapamycin treatment or genetically induced decreases in mTORC1 activity in podocytes were also investigated $(8,9)$. Loss of mTORC1 in utero in podocytes resulted in proteinuria preceding podocyte loss, whereas adult loss of mTORC1 had no significant effect (8).
Further, adding rapamycin early prevented renal injury caused by mTORC1 activation (9). However, progression of sclerosis was not affected if rapamycin was given when injury induced by mTORC1 activation in podocytes was already moderate (9). These results mirror those seen in previous studies of potential regression of sclerosis, where glomeruli with more severe sclerosis could not be rescued with delayed intervention with high-dose angiotensin inhibition (13). In clinical practice, patients typically present with late-stage disease, and the current studies suggest that mTOR manipulation may not be effective at this stage. However, even very early inhibition of mTOR in patients with presumed early diabetic injury, namely those receiving pancreas transplant for diabetes without kidney transplant, resulted in proteinuria, perhaps because hypertrophy was already present (10). Recent data support a two-hit model of podocyte injury for sclerosis, with ongoing filtration and direct injury effecting podocyte loss and sclerosis and a spreading of injury from podocytes initially sustaining the insult to intact podocytes $(14,15)$.

\section{Varying genetic susceptibility to $\mathrm{mTORC}$-induced injury}

Interestingly, the consequences of genetic manipulation of podocyte mTOR activity were strain- and age-dependent $(8,9)$. mTORC1 appears to contribute to development of normal-size glomeruli and podocytes. Decreased mTORC1 activity resulted in glomerular injury in young mice and in 
the susceptible ICR strain but not in adult mice or the resistant C57BL/6 strain (8). Similarly, in humans, the loss of one kidney has minimal consequences in healthy adults, whereas children with nephrectomy at a young age have markedly increased incidence of albuminuria and hypertension at follow-up $(16,17)$. The incidence of CKD, particularly hypertension-associated or due to FSGS, is markedly increased in African Americans compared with those of European descent (17). Various factors have been proposed, including an APOL1 gene variant and low birth weight linked to low nephron number $(17,18)$. Whether mTORC1 alterations could influence human nephron development and also later susceptibility to additional injuries is not known. Additional signaling pathways such as the Notch, PPAR $\gamma$, and calcineurin pathways also modulate the podocyte and its injury responses (19-21). Interactions of these pathways with mTORC actions in podocytes are not yet known.

\section{Clinical implications moving forward}

The challenge is to identify specific disease stages for beneficial manipulation of mTOR pathway activity. Taken together, the work of Gödel et al. (8) and Inoki et al. (9) demonstrate that too little or too much mTOR activity is deleterious to the podocyte, analogous to the fine-tuned balance of VEGF actions on the glomerulus. The findings add yet another complex dimension to the understanding of the dynamic regula- tion of the podocyte. Further in-depth examination of mechanisms in experimental models that faithfully capture key elements of human disease counterparts, and ultimately translation to human conditions, will be necessary.

\section{Acknowledgments}

This work was supported in part by NIH grants NIDDK R01DK56942 and P50 DK44757.

Address correspondence to: Agnes B. Fogo, MCN C3310, Department of Pathology, Vanderbilt University Medical Center, Nashville, Tennessee 37232, USA. Phone: 615.322.3070; Fax: 615.343.7023; E-mail: agnes.fogo@vanderbilt.edu.

1. Mathieson PW. Update on the podocyte. Curr Opin Nephrol Hypertens. 2009;18(3):206-211.

2 . Ohse $\mathrm{T}$, et al. The enigmatic parietal epithelial cell is finally getting noticed: a review. Kidney Int. 2009;76(12):1225-1238.

3. Fatima H, Moeller MJ, Smeets B, Yang H-C, Fogo AB. Parietal epithelial cell activation distinguishes early recurrence of FSGS in the transplant from minimal change disease. (abstract 1460) Lab Invest. 2011;91(1):343A.

4. Smeets B, Dijkman HB, Wetzels JF, Steenbergen EJ. Lessons from studies on focal segmental glomerulosclerosis: an important role for parietal epithelial cells? J Pathol. 2006;210(3):263-272.

5. Fioretto P, Steffes MW, Sutherland DE, Goetz FC, Mauer M. Reversal of lesions of diabetic nephropathy after pancreas transplantation. N Engl J Med. 1998; 339(2):69-75

6. Alpers CE, Hudkins KL. Mouse models of diabetic nephropathy. Curr Opin Nephrol Hypertens. 2011; 20(3):278-284.

7. Fogo AB. Can glomerulosclerosis be reversed? Nat
Clin Pract Nephrol. 2006;2(6):290-291.

8. Gödel M, et al. Role of mTOR in podocyte function and diabetic nephropathy in humans and mice. J Clin Invest. 2011;121(6):2197-2209.

9. Inoki $\mathrm{K}$, et al. mTORC1 activation in podocytes is a critical step in the development of diabetic nephropathy in mice. J Clin Invest. 2011;121(6):2181-2196.

10. Huber TB, Walz G, Kuehn EW. mTOR and rapamycin in the kidney: signaling and therapeutic implications beyond immunosuppression. Kidney Int. 2011;79(5):502-511.

11. Yamaguchi Y, et al. Epithelial-mesenchymal transition as a potential explanation for podocyte depletion in diabetic nephropathy. Am J Kidney Dis. 2009;54(4):653-664.

12. Yang $H$, Fogo AB. Cell senescence in the aging kidney. J Am Soc Nephrol. 2010;21(9):1436-1439.

13. Ikoma M, Kawamura T, Kakinuma Y, Fogo A, Ichikawa I. Cause of variable therapeutic efficiency of angiotensin converting enzyme inhibitor on glomerular lesions. Kidney Int. 1991;40(2):195-202.

14. Matsusaka T, Kobayashi K, Kon V, Pastan I, Fogo AB, Ichikawa I. Glomerular sclerosis is prevented during urinary tract obstruction due to podocyte protection. Am J Physiol Renal Physiol. 2011;300(3):F792-F800.

15. Matsusaka T, et al. Study of genetically engineered chimeric glomeruli revealed podocyte damage damages other podocytes. J Am Soc Nephrol. In press.

16. Di Tullio MT, et al. Compensatory hypertrophy and progressive renal damage in children nephrectomized for Wilms' tumor. Med Pediatr Oncol. 1996; 26(5):325-328.

17. Marcantoni C, Fogo AB. A perspective on arterionephrosclerosis: from pathology to potential pathogenesis. J Nephrol. 2007;20(5):518-524.

18. Genovese G, et al. Association of trypanolytic ApoL1 variants with kidney disease in African Americans. Science. 2010;329(5993):841-845.

19. Murea M, et al. Expression of Notch pathway proteins correlates with albuminuria, glomerulosclerosis, and renal function. Kidney Int. 2010;78(5):514-522.

20. Kanjanabuch T, et al. PPAR-gamma agonist protects podocytes from injury. Kidney Int. 2007; 71(12):1232-1239.

21. Faul C, et al. The actin cytoskeleton of kidney podocytes is a direct target of the antiproteinuric effect of cyclosporine A. Nat Med. 2008;14(9):931-938.

\title{
Spotlight on childhood blindness
}

\author{
José-Alain Sahel1,2,3,4,5,6
}

${ }^{1}$ INSERM, U968, Paris, France. ${ }^{2}$ Université Pierre et Marie Curie-Paris6, UM80, Institut de la Vision, Paris, France. ${ }^{3}$ CNRS, UMR_7210, Paris, France. ${ }^{4}$ Centre Hospitalier National d'Ophtalmologie des Quinze-Vingts, INSERM-DHOS CIC 503, Paris, France. ${ }^{5}$ Fondation Ophtalmologique Adolphe de Rothschild, Paris, France. ${ }^{6}$ Institute of Ophthalmology, University College of London, London, United Kingdom.

\begin{abstract}
Leber congenital amaurosis (LCA) is a rare disease that severely affects vision in early life. It is characterized by genetic and clinical heterogeneity due to complex and not fully understood pathogenetic mechanisms. It is also now widely known as a disease model for gene therapy. In this issue of the JCI, two independent research groups report valuable new data on LCA. Specifically, they provide important insights into the pathophysiological mechanisms of LCA and offer strong hope that the outcome of gene therapy for retinal degenerative diseases will be successful.
\end{abstract}

Conflict of interest: The author receives research support and income from Fovea-Pharmaceuticals-Sanofi. Citation for this article: J Clin Invest. doi:10.1172/ JCI58300.

\section{Highlights of LCA}

Leber congenital amaurosis (LCA) is a rare retinal dystrophy with a prevalence at birth of approximately 1 in 80,000 (1). It is most often inherited in an autosomal-recessive manner. It typically becomes evident in the first year of life, with poor visual function often accompanied by nystagmus, sluggish or near-absent pupillary responses, photophobia, high hyperopia, and keratoconus. Profound visual impairment is usually present from birth.

LCA is caused by mutations in any one of at least 15 genes (2), and this accounts in part for its heterogeneous clinical presentation. To date, more than 400 mutations have been identified. Together, they account 